\title{
Application of Parseval's Theorem on Evaluating Some Definite Integrals
}

\author{
Chii-Huei Yu* \\ Department of Management and Information, Nan Jeon University of Science and Technology, Tainan City, Taiwan \\ *Corresponding author: chiihuei@nju.edu.tw
}

Received December 08, 2013; Revised January 09, 2014; Accepted January 16, 2014

\begin{abstract}
This paper uses the mathematical software Maple for the auxiliary tool to study six types of definite integrals. We can determine the infinite series forms of these definite integrals by using Parseval's theorem. On the other hand, we provide some definite integrals to do calculation practically. The research methods adopted in this study involved finding solutions through manual calculations and verifying these solutions using Maple.
\end{abstract}

Keywords: definite integrals, infinite series forms, Parseval's theorem, Maple

Cite This Article: Chii-Huei Yu, "Application of Parseval's Theorem on Evaluating Some Definite Integrals." Turkish Journal of Analysis and Number Theory 2, no. 1 (2014): 1-5. doi: 10.12691/tjant-2-1-1.

\section{Introduction}

As information technology advances, whether computers can become comparable with human brains to perform abstract tasks, such as abstract art similar to the paintings of Picasso and musical compositions similar to those of Mozart, is a natural question. Currently, this appears unattainable. In addition, whether computers can solve abstract and difficult mathematical problems and develop abstract mathematical theories such as those of mathematicians also appears unfeasible. Nevertheless, in seeking for alternatives, we can study what assistance mathematical software can provide. This study introduces how to conduct mathematical research using the mathematical software Maple. The main reasons of using Maple in this study are its simple instructions and ease of use, which enable beginners to learn the operating techniques in a short period. By employing the powerful computing capabilities of Maple, difficult problems can be easily solved. Even when Maple cannot determine the solution, problem-solving hints can be identified and inferred from the approximate values calculated and solutions to similar problems, as determined by Maple. For this reason, Maple can provide insights into scientific research.

In calculus and engineering mathematics courses, we learnt many methods to solve the integral problems including change of variables method, integration by parts method, partial fractions method, trigonometric substitution method, and so on. In this paper, we study the following six types of definite integrals which are not easy to obtain their answers using the methods mentioned above.

$$
\int_{0}^{2 \pi} \sinh ^{2}(r \cos x) \cdot \cos ^{2}(r \sin x) d x
$$

$$
\begin{aligned}
& \int_{0}^{2 \pi} \cosh ^{2}(r \cos x) \cdot \sin ^{2}(r \sin x) d x \\
& \int_{0}^{2 \pi}\left[\sinh ^{2}(r \cos x)+\sin ^{2}(r \sin x)\right] d x \\
& \int_{0}^{2 \pi} \cosh ^{2}(r \cos x) \cdot \cos ^{2}(r \sin x) d x \\
& \int_{0}^{2 \pi} \sinh ^{2}(r \cos x) \cdot \sin ^{2}(r \sin x) d x \\
& \int_{0}^{2 \pi}\left[\cosh ^{2}(r \cos x)+\cos ^{2}(r \sin x)\right] d x
\end{aligned}
$$

where $r$ is any real number. We can obtain the infinite series forms of these definite integrals by using Parseval's theorem; these are the major results of this paper (i.e., Theorems 1 and 2). As for the study of related integral problems can refer to [1-17]. On the other hand, we propose some definite integrals to do calculation practically. The research methods adopted in this study involved finding solutions through manual calculations and verifying these solutions by using Maple. This type of research method not only allows the discovery of calculation errors, but also helps modify the original directions of thinking from manual and Maple calculations. For this reason, Maple provides insights and guidance regarding problem-solving methods.

\section{Main Results}

Firstly, we introduce a notation and a definition and some formulas used in this article.

\subsection{Notation}

Let $z=a+i b$ be a complex number, where $i=\sqrt{-1}$, $a, b$ are real numbers. We denote $a$ the real part of $z$ by $\operatorname{Re}(z)$, and $b$ the imaginary part of $z$ by $\operatorname{Im}(z)$. 


\subsection{Definition}

Suppose $f(x)$ is a continuous function defined on $[0,2 \pi]$, then the Fourier series expansion of $f(x)$ is $\frac{1}{2} a_{0}+\sum_{k=1}^{\infty}\left(a_{k} \cos k x+b_{k} \sin k x\right)$ where $a_{0}=\frac{1}{\pi} \int_{0}^{2 \pi} f(x) d x \quad, \quad$ and $\quad a_{k}=\frac{1}{\pi} \int_{0}^{2 \pi} f(x) \cos k x d x$, $b_{k}=\frac{1}{\pi} \int_{0}^{2 \pi} f(x) \sin k x d x$ for all positive integers $k$.

\subsection{Formulas}

\subsubsection{Euler's Formula}

$e^{i x}=\cos x+i \sin x$, where $x$ is any real number.

\subsubsection{DeMoivre's Formula}

$(\cos x+i \sin x)^{n}=\cos n x+i \sin n x$, where $n$ is any integer, and $x$ is any real number.

\subsubsection{Taylor Series Expansion of Hyperbolic Sine} Function ([18])

$\sinh (z)=\sum_{k=0}^{\infty} \frac{z^{2 k+1}}{(2 k+1) !}$, where $z$ is any complex number.

\subsubsection{Taylor Series Expansion of Hyperbolic Cosine} Function ([18])

$\cosh (z)=\sum_{k=0}^{\infty} \frac{z^{2 k}}{(2 k) !}$, where $z$ is any complex number.

Next, we introduce an important theorem used in this study.

\subsection{Parseval's Theorem ([19])}

If $f(x)$ is a continuous function defined on $[0,2 \pi]$, and $f(0)=f(2 \pi)$. If the Fourier series expansion of $f(x) \quad$ is $\quad \frac{1}{2} a_{0}+\sum_{k=1}^{\infty}\left(a_{k} \cos k x+b_{k} \sin k x\right) \quad$, then $\frac{1}{\pi} \int_{0}^{2 \pi} f^{2}(x) d x=\frac{a_{0}^{2}}{2}+\sum_{k=1}^{\infty}\left(a_{k}^{2}+b_{k}^{2}\right)$.

Before deriving the first major result of this paper, we need a lemma.

\subsection{Lemma 1}

Suppose $p, q$ are any real numbers. Then

$$
\begin{gathered}
\sinh (p+i q)=\sinh p \cdot \cos q+i \cosh p \cdot \sin q \\
\sinh ^{2} p \cdot \cos ^{2} q+\cosh ^{2} p \cdot \sin ^{2} q=\sinh ^{2} p+\sin ^{2} q
\end{gathered}
$$

Proof $\sinh (p+i q)$

$$
\begin{aligned}
& =\frac{1}{2}\left[e^{p+i q}-e^{-(p+i q)}\right] \\
& =\frac{1}{2}\left[e^{p}(\cos q+i \sin q)-e^{-p}(\cos q-i \sin q)\right]
\end{aligned}
$$

$=\frac{1}{2}\left(e^{p}-e^{-p}\right) \cos q+i \frac{1}{2}\left(e^{p}+e^{-p}\right) \sin q$

$=\sinh p \cdot \cos q+i \cosh p \cdot \sin q$

And

$$
\begin{aligned}
& \sinh ^{2} p \cdot \cos ^{2} q+\cosh ^{2} p \cdot \sin ^{2} q \\
= & \sinh ^{2} p \cdot\left(1-\sin ^{2} q\right)+\cosh ^{2} p \cdot \sin ^{2} q \\
= & \sinh ^{2} p+\sin ^{2} q
\end{aligned}
$$

Next, we determine the infinite series forms of the definite integrals (1), (2) and (3).

\subsection{Theorem 1}

Suppose $r$ is any real number. Then the definite integrals

$$
\begin{aligned}
& \int_{0}^{2 \pi} \sinh ^{2}(r \cos x) \cdot \cos ^{2}(r \sin x) d x \\
= & \pi \cdot \sum_{k=0}^{\infty} \frac{r^{4 k+2}}{[(2 k+1) !]^{2}} \\
& \int_{0}^{2 \pi} \cosh ^{2}(r \cos x) \cdot \sin ^{2}(r \sin x) d x \\
= & \pi \cdot \sum_{k=0}^{\infty} \frac{r^{4 k+2}}{[(2 k+1) !]^{2}} \\
& \int_{0}^{2 \pi}\left[\sinh ^{2}(r \cos x)+\sin ^{2}(r \sin x)\right] d x \\
= & 2 \pi \cdot \sum_{k=0}^{\infty} \frac{r^{4 k+2}}{[(2 k+1) !]^{2}}
\end{aligned}
$$

\section{Proof Because}

$\sinh (r \cos x) \cdot \cos (r \sin x)$

$$
\begin{aligned}
& =\operatorname{Re}\left[\sinh \left(r e^{i x}\right)\right] \quad(B y(7)) \\
& =\operatorname{Re}\left[\sum_{k=0}^{\infty} \frac{\left(r e^{i x}\right)^{2 k+1}}{(2 k+1) !}\right] \\
& =\operatorname{Re}\left[\sum_{k=0}^{\infty} \frac{r^{2 k+1} e^{i(2 k+1) x}}{(2 k+1) !}\right] \\
& =\sum_{k=0}^{\infty} \frac{r^{2 k+1}}{(2 k+1) !} \cos (2 k+1) x
\end{aligned}
$$

It follows that

$$
\begin{aligned}
& \int_{0}^{2 \pi} \sinh ^{2}(r \cos x) \cdot \cos ^{2}(r \sin x) d x \\
= & \pi \cdot \sum_{k=0}^{\infty} \frac{r^{4 k+2}}{[(2 k+1) !]^{2}}
\end{aligned}
$$

(Using (12) and Parseval's theorem)

Similarly, because

$\cosh (r \cos x) \cdot \sin (r \sin x)$

$=\operatorname{Im}\left[\sinh \left(r e^{i x}\right)\right] \quad(B y(7))$

$=\operatorname{Im}\left[\sum_{k=0}^{\infty} \frac{\left(r e^{i x}\right)^{2 k+1}}{(2 k+1) !}\right]$ 
$=\sum_{k=0}^{\infty} \frac{r^{2 k+1}}{(2 k+1) !} \sin (2 k+1) x$

It follows that

$$
\begin{aligned}
& \int_{0}^{2 \pi} \cosh ^{2}(r \cos x) \cdot \sin ^{2}(r \sin x) d x \\
= & \pi \cdot \sum_{k=0}^{\infty} \frac{r^{4 k+2}}{[(2 k+1) !]^{2}}
\end{aligned}
$$

(Using (13) and Parseval's theorem)

On the other hand, from the summation of (9) and (10) and using (8), we obtain

$$
\begin{aligned}
& \int_{0}^{2 \pi}\left[\sinh ^{2}(r \cos x)+\sin ^{2}(r \sin x)\right] d x \\
= & 2 \pi \cdot \sum_{k=0}^{\infty} \frac{r^{4 k+2}}{[(2 k+1) !]^{2}}
\end{aligned}
$$

Before deriving the second major result of this study, we also need a lemma.

\subsection{Lemma 2}

Suppose $p, q$ are any real numbers. Then

$$
\begin{aligned}
& \quad \cosh (p+i q)=\cosh p \cdot \cos q+i \sinh p \cdot \sin q \\
& \quad \cosh ^{2} p \cdot \cos ^{2} q+\sinh ^{2} p \cdot \sin ^{2} q=\sinh ^{2} p+\cos \\
& \text { Proof } \cosh (p+i q) \\
& =\frac{1}{2}\left[e^{p+i q}+e^{-(p+i q)}\right] \\
& =\frac{1}{2}\left[e^{p}(\cos q+i \sin q)+e^{-p}(\cos q-i \sin q)\right] \\
& =\frac{1}{2}\left(e^{p}+e^{-p}\right) \cos q+i \frac{1}{2}\left(e^{p}-e^{-p}\right) \sin q \\
& =\cosh p \cdot \cos q+i \sinh p \cdot \sin q
\end{aligned}
$$

$\cosh ^{2} p \cdot \cos ^{2} q+\sinh ^{2} p \cdot \sin ^{2} q=\sinh ^{2} p+\cos ^{2} q(15)$

And

$$
\begin{aligned}
& \cosh ^{2} p \cdot \cos ^{2} q+\sinh ^{2} p \cdot \sin ^{2} q \\
= & \left(1+\sinh ^{2} p\right) \cdot \cos ^{2} q+\sinh ^{2} p \cdot \sin ^{2} q \\
= & \sinh ^{2} p+\cos ^{2} q
\end{aligned}
$$

Finally, we find the infinite series forms of the definite integrals (4), (5) and (6).

\subsection{Theorem 2}

Suppose $r$ is any real number. Then the definite integrals

$$
\begin{aligned}
& \int_{0}^{2 \pi} \cosh ^{2}(r \cos x) \cdot \cos ^{2}(r \sin x) d x \\
= & 2 \pi+\pi \sum_{k=1}^{\infty} \frac{r^{4 k}}{[(2 k) !]^{2}} \\
& \int_{0}^{2 \pi} \sinh ^{2}(r \cos x) \cdot \sin ^{2}(r \sin x) d x \\
= & \pi \cdot \sum_{k=1}^{\infty} \frac{r^{4 k}}{[(2 k) !]^{2}}
\end{aligned}
$$

$$
\begin{aligned}
& \int_{0}^{2 \pi}\left[\sinh ^{2}(r \cos x)+\cos ^{2}(r \sin x)\right] d x \\
= & 2 \pi+2 \pi \cdot \sum_{k=1}^{\infty} \frac{r^{4 k}}{[(2 k) !]^{2}}
\end{aligned}
$$

Proof Because

$$
\cosh (r \cos x) \cdot \cos (r \sin x)
$$

$=\operatorname{Re}\left[\cosh \left(r e^{i x}\right)\right] \quad(B y(14))$

$=\operatorname{Re}\left[\sum_{k=0}^{\infty} \frac{\left(r e^{i x}\right)^{2 k}}{(2 k) !}\right] \quad$ (By Formula 2.3.4.)

$=1+\sum_{k=1}^{\infty} \frac{r^{2 k}}{(2 k) !} \cos 2 k x$

(By DeMoivre's formula and Euler's formula)

Using (19) and Parseval's theorem, we have

$$
\begin{aligned}
& \int_{0}^{2 \pi} \cosh ^{2}(r \cos x) \cdot \cos ^{2}(r \sin x) d x \\
= & 2 \pi+\pi \sum_{k=1}^{\infty} \frac{r^{4 k}}{[(2 k) !]^{2}}
\end{aligned}
$$

Similarly, because

$\sinh (r \cos x) \cdot \sin (r \sin x)$

$=\operatorname{Im}\left[\cosh \left(r e^{i x}\right)\right]$

$=\operatorname{Im}\left[\sum_{k=0}^{\infty} \frac{\left(r e^{i x}\right)^{2 k}}{(2 k) !}\right]$

$=\sum_{k=1}^{\infty} \frac{r^{2 k}}{(2 k) !} \sin 2 k x$

It follows that

$$
\begin{aligned}
& \int_{0}^{2 \pi} \sinh ^{2}(r \cos x) \cdot \sin ^{2}(r \sin x) d x \\
= & \pi \cdot \sum_{k=1}^{\infty} \frac{r^{4 k}}{[(2 k) !]^{2}}
\end{aligned}
$$

(By (20) and Parseval's theorem)

From the summation of (16) and (17) and using (15), we have

$$
\begin{aligned}
& \int_{0}^{2 \pi}\left[\sinh ^{2}(r \cos x)+\cos ^{2}(r \sin x)\right] d x \\
= & 2 \pi+2 \pi \cdot \sum_{k=1}^{\infty} \frac{r^{4 k}}{[(2 k) !]^{2}}
\end{aligned}
$$

\section{Examples}

In the following, for the six types of definite integrals in this study, we provide some definite integrals and use Theorems 1 and 2 to determine their infinite series forms. On the other hand, we employ Maple to calculate the approximations of these definite integrals and their solutions for verifying our answers.

\subsection{Example 1}

Taking $r=7$ into (9), we obtain the definite integral 


$$
\begin{aligned}
& \int_{0}^{2 \pi} \sinh ^{2}(7 \cos x) \cdot \cos ^{2}(7 \sin x) d x \\
= & \pi \cdot \sum_{k=0}^{\infty} \frac{7^{4 k+2}}{[(2 k+1) !]^{2}}
\end{aligned}
$$

Next, we use Maple to verify the correctness of (21). $>\operatorname{evalf}\left(\operatorname{int}\left((\sinh (7 * \cos (\mathrm{x})))^{\wedge} 2 *(\cos (7 * \sin (\mathrm{x})))^{\wedge} 2, \mathrm{x}=0 . .2 * \mathrm{Pi}\right.\right.$ )$, 18)$;

$$
2.03289934187665893 \cdot 10^{5}
$$

$>\operatorname{evalf}\left(\mathrm{Pi}^{*} \operatorname{sum}\left(7^{\wedge}\left(4^{*} \mathrm{k}+2\right) /((2 * \mathrm{k}+1) !)^{\wedge} 2, \mathrm{k}=0\right.\right.$..infinity $\left.), 18\right)$;

$$
2.03289934187665894 \cdot 10^{5}
$$

Also, let $r=\sqrt{3}$ in (10), we have

$$
\begin{aligned}
& \int_{0}^{2 \pi} \cosh ^{2}(\sqrt{3} \cos x) \cdot \sin ^{2}(\sqrt{3} \sin x) d x \\
= & \pi \cdot \sum_{k=0}^{\infty} \frac{(\sqrt{3})^{4 k+2}}{[(2 k+1) !]^{2}}
\end{aligned}
$$

$>\operatorname{evalf}\left(\operatorname{int}((\cosh (\operatorname{sqrt}(3) * \cos (\mathrm{x}))))^{\wedge} 2 *\left(\sin \left(\operatorname{sqrt}(3)^{*} \sin (\mathrm{x})\right)\right)^{\wedge}\right.$ $2, \mathrm{x}=0 . .2 * \mathrm{Pi}), 18)$;

\subsection{6}

$>\operatorname{evalf}\left(\operatorname{Pi} * \operatorname{sum}((\operatorname{sqrt}(3)))^{\wedge}(4 * \mathrm{k}+2) /((2 * \mathrm{k}+1) !)^{\wedge} 2, \mathrm{k}=0\right.$.. infinity), 18);

\subsection{5}

Finally, if $r=5 / 3$ in (11), then

$$
\begin{aligned}
& \int_{0}^{2 \pi}\left[\sinh ^{2}\left(\frac{5}{3} \cos x\right)+\sin ^{2}\left(\frac{5}{3} \sin x\right)\right] d x \\
= & 2 \pi \cdot \sum_{k=0}^{\infty} \frac{(5 / 3)^{4 k+2}}{[(2 k+1) !]^{2}}
\end{aligned}
$$

$>\operatorname{evalf}\left(\operatorname{int}((\sinh (5 / 3 * \cos (\mathrm{x}))))^{\wedge} 2+(\sin (5 / 3 * \sin (\mathrm{x})))\right)^{\wedge} 2, \mathrm{x}=0 . .2$ *Pi), 18);

\subsection{1}

$>\operatorname{evalf}\left(2 * \mathrm{Pi}^{*} \operatorname{sum}\left((5 / 3)^{\wedge}(4 * \mathrm{k}+2) /((2 * \mathrm{k}+1) !)^{\wedge} 2, \mathrm{k}=0\right.\right.$. infinity), 18);

\subsection{0}

\subsection{Example 2}

Taking $r=9$ into (16), then the definite integral

$$
\begin{aligned}
& \int_{0}^{2 \pi} \cosh ^{2}(9 \cos x) \cdot \cos ^{2}(9 \sin x) d x \\
= & 2 \pi+\pi \sum_{k=1}^{\infty} \frac{9^{4 k}}{[(2 k) !]^{2}}
\end{aligned}
$$

$>$ evalf $\left(\operatorname{int}\left((\cosh (9 * \cos (\mathrm{x})))^{\wedge} 2 *(\cos (9 * \sin (\mathrm{x})))^{\wedge} 2, \mathrm{x}=0 . .2 * \mathrm{Pi}\right.\right.$ )$, 18)$;

\section{$9.76786250967196351 \cdot 10^{6}$}

$>\operatorname{evalf}\left(2 * \mathrm{Pi}+\mathrm{Pi}^{*} \operatorname{sum}\left(9^{\wedge}(4 * \mathrm{k}) /((2 * \mathrm{k}) !)^{\wedge} 2, \mathrm{k}=1\right.\right.$...infinity $\left.), 18\right)$;

$$
9.76786250967196350 \cdot 10^{6}
$$

In addition, let $r=\sqrt{11}$ in (17), then

$$
\begin{aligned}
& \int_{0}^{2 \pi} \sinh ^{2}(\sqrt{11} \cos x) \cdot \sin ^{2}(\sqrt{11} \sin x) d x \\
= & \pi \cdot \sum_{k=1}^{\infty} \frac{(\sqrt{11})^{4 k}}{[(2 k) !]^{2}}
\end{aligned}
$$

$>$ evalf $\left(\operatorname{int}\left((\sinh (\operatorname{sqrt}(11) * \cos (\mathrm{x})))^{\wedge} 2 *(\sin (\operatorname{sqrt}(11) * \sin (\mathrm{x})))\right.\right.$ $\wedge 2, \mathrm{x}=0 . .2 * \mathrm{Pi}), 18)$;

\subsection{7}

$>\operatorname{evalf}\left(\mathrm{Pi}^{*} \operatorname{sum}\left((\operatorname{sqrt}(11))^{\wedge}(4 * \mathrm{k}) /((2 * \mathrm{k}) !)^{\wedge} 2, \mathrm{k}=1\right.\right.$..infinity $)$, 18);

\subsection{7}

Finally, if $r=10 / 7$ in (18), we have

$$
\begin{aligned}
& \int_{0}^{2 \pi}\left[\sinh ^{2}\left(\frac{10}{7} \cos x\right)+\cos ^{2}\left(\frac{10}{7} \sin x\right)\right] d x \\
= & 2 \pi+2 \pi \cdot \sum_{k=1}^{\infty} \frac{(10 / 7)^{4 k}}{[(2 k) !]^{2}}
\end{aligned}
$$

$>\operatorname{evalf}\left(\operatorname{int}\left((\sinh (10 / 7 * \cos (\mathrm{x})))^{\wedge} 2+(\cos (10 / 7 * \sin (\mathrm{x})))^{\wedge} 2, \mathrm{x}=\right.\right.$ $0 . .2 * \mathrm{Pi}), 18)$;

\subsection{0}

$>\operatorname{evalf}\left(2 * \mathrm{Pi}+2 * \mathrm{Pi}^{*} \operatorname{sum}\left((10 / 7)^{\wedge}(4 * \mathrm{k}) /((2 * \mathrm{k}) !)^{\wedge} 2, \mathrm{k}=1 .\right.\right.$. infinity), 18);

\subsection{9}

\section{Conclusion}

In this paper, we provide a new technique to determine some definite integrals. We hope this technique can be applied to solve another definite integral problems. On the other hand, the Parseval's theorem plays a significant role in the theoretical inferences of this study. In fact, the applications of this theorem are extensive, and can be used to easily solve many difficult problems; we endeavor to conduct further studies on related applications. In addition, Maple also plays a vital assistive role in problem-solving. In the future, we will extend the research topic to other calculus and engineering mathematics problems and solve these problems by using Maple. These results will be used as teaching materials for Maple on education and research to enhance the connotations of calculus and engineering mathematics.

\section{References}

[1] C. Oster, "Limit of a definite integral," SIAM Review, vol. 33, no. 1, pp. 115-116, 1991.

[2] A. A. Adams, H. Gottliebsen, S. A. Linton, and U. Martin, "Automated theorem proving in support of computer algebra: symbolic definite integration as a case study," Proceedings of the 1999 International Symposium on Symbolic and Algebraic Computation, pp. 253-260, Canada, 1999.

[3] M. A. Nyblom, "On the evaluation of a definite integral involving nested square root functions," Rocky Mountain Journal of Mathematics, vol. 37, no. 4, pp. 1301-1304, 2007.

[4] C. -H. Yu, "Using Maple to study two types of integrals," International Journal of Research in Computer Applications and Robotics, vol. 1, issue. 4, pp. 14-22, 2013.

[5] C. -H. Yu, "Solving some integrals with Maple," International Journal of Research in Aeronautical and Mechanical Engineering, vol. 1, issue. 3, pp. 29-35, 2013. 
[6] C. -H. Yu, "A study on integral problems by using Maple," International Journal of Advanced Research in Computer Science and Software Engineering, vol. 3, issue. 7, pp. 41-46, 2013.

[7] C. - H. Yu, "Evaluating some integrals with Maple, "International Journal of Computer Science and Mobile Computing, vol. 2, issue. 7, pp. 66-71, 2013.

[8] C.-H. Yu, "Application of Maple on evaluation of definite integrals," Applied Mechanics and Materials, vols. 479-480, pp. 823-827, 2013

[9] C. -H. Yu, "A study of some integral problems using Maple," Mathematics and Statistics, vol. 2, no. 1, pp. 1-5, 2014.

[10] C.-H. Yu, "Application of Maple on the integral problems," Applied Mechanics and Materials, vols. 479-480, pp. 849-854, 2013.

[11] C.-H. Yu, "Application of Maple on the integral problem of some type of rational functions," Proceedings of the Annual Meeting and Academic Conference for Association of IE, D357-D362, 2012.

[12] C. -H. Yu, "Using Maple to study the integrals of trigonometric functions," Proceedings of the 6th IEEE/International Conference on Advanced Infocomm Technology, no. 00294, 2013.
[13] C.-H. Yu, "Application of Maple on some type of integral problem," Proceedings of the Ubiquitous-Home Conference 2012, pp. 206-210, 2012.

[14] C. -H. Yu, "A study of the integrals of trigonometric functions with Maple," Proceedings of the Institute of Industrial Engineers Asian Conference 2013, Springer, vol. 1, pp. 603-610, 2013.

[15] C.-H. Yu, "Application of Maple on some integral problems, "Proceedings of the International Conference on Safety \& Security Management and Engineering Technology 2012, pp. 290-294, 2012.

[16] C.-H. Yu, "Application of Maple on evaluating the closed forms of two types of integrals," Proceedings of the 17th Mobile Computing Workshop, ID16, 2012.

[17] C.-H. Yu, "Application of Maple: taking two special integral problems as examples," Proceedings of the 8th International Conference on Knowledge Community, pp. 803-811, 2012.

[18] Hyperbolic functions, online available from http://en.wikipedia.org/wiki/Hyperbolic_function.

[19] D. V. Widder, Advanced calculus, 2nd ed., Prentice-Hall, New Jersey, p 428, 1961. 